\title{
A Wide Dynamic Range CMOS Image Sensor with Resistance to High Temperatures
}

\author{
Koichi Mizobuchi ${ }^{1}$, Satoru Adachi ${ }^{1}$, Tomokazu Yamashita ${ }^{1}$, Seiichiro Okamura ${ }^{1}$, \\ Hiromichi Oshikubo ${ }^{1}$, Nana Akahane ${ }^{2}$ and Shigetoshi Sugawa ${ }^{2}$ \\ ${ }^{1}$ DISP Development, Texas Instruments Japan, 2350 Kihara, Miho, Inashiki, Ibaraki 300-0496, Japan \\ Tel: +81-29-880-4066, Fax: +81-29-880-3242 \\ ${ }^{2}$ Gradute School of Engineering, Tohoku University, 6-6-11 Aramaki, Aza-Aoba, Aoba-ku, Sendai, 980-8579, Japan \\ Tel: +81-22-795-4833, Fax:+81-22-795-4834 \\ E-mail: \{mizo, chiro, cdty, iapo, hiromichi-oshikubo\}@ti.com, \{nana, sugawa\}@fff.niche.tohoku.ac.jp
}

\begin{abstract}
A $1 / 4$ inch VGA $5.6 \mu \mathrm{m}$ pixel pitch wide dynamic range (WDR) CMOS image sensor with resistance to high temperatures has been developed using a very-low-dark-current front-end of line (VLDC FEOL), a metal hermetic seal package and/or the inorganic cap layer to suppress the degradation of the spectra response of the on-chip micro lens and color filter (OCML/OCCF). The dark current is reduced to 175 $\mathrm{e}^{-} / \mathrm{sec}-$ pixel at $85^{\circ} \mathrm{C}\left(25 \mathrm{e} / \mathrm{sec}-\right.$ pixel at $\left.60^{\circ} \mathrm{C}\right)$ by the use of VLDC FEOL. Sensor chips with no cap and an inorganic cap onto the OCML were assembled into a metal seal package and a conventional package respectively. They were exposed to a $150^{\circ} \mathrm{C} / 500$ hours thermal-stress test in the air. No degradation of the spectra response in any of $\mathrm{R} / \mathrm{G} / \mathrm{B}$ pixels for both samples is observed after the thermal stress test. The sample images captured by the WDR CMOS image sensor with and without the thermal stress show no significant degradation in image quality up to $85^{\circ} \mathrm{C}$. The image sensing performance results in a low noise and huge effective saturation voltage. The dynamic range is extended to $93 \mathrm{~dB}$.
\end{abstract}

\section{Introduction}

A wide-dynamic-range (WDR) CMOS image sensor using a lateral overflow integration capacitor in each pixel demonstrated over-100 dB dynamic range (DR) in single exposure and over- $200 \mathrm{~dB}$ DR in multiple exposures plus the current readout operation, keeping a high sensitivity and a high $\mathrm{S} / \mathrm{N}$ ratio [1-4]. Automotive applications, for which this sensor best suits, require an operation temperature typically ranging from -40 to $85^{\circ} \mathrm{C}$ or much higher. Security and medical applications also desire the extension of operation temperatures. In these hard conditions, especially at very high temperatures, the increase of dark current shot noise requires careful attention. Thermal decomposition of organic materials in the sensor chip and/or the package is also an issue. Several papers have reported dark current reduction approaches $[5,6]$. However a temperature-resistant image sensing solution has not been clearly reported as the discussion has just started [7]. This paper describes a WDR CMOS image sensor with resistance to high temperatures using a very-low-dark-current front-end of line (VLDC FEOL) and an inorganic cap on the on-chip micro lens (OCML) as well as a metal hermetic seal package to suppress thermal decomposition of both the OCML and the on-chip color filter (OCCF).

\section{Dynamic Range Enhancement}

Fig. 1 shows a pixel schematic circuit diagram adopted for the wide dynamic range solution. The pixel circuit consists of a fully depleted photodiode (PD), a floating diffusion to convert the charge to the voltage (FD), a charge transfer switch (M1), an overflow photoelectron integration capacitor (CS), a switch between the floating diffusion FD and the overflow capacitor CS (M3), a reset switch (M2), a source follower amplifier (M4) and a pixel select switch (M5). The basic concept in this pixel circuit is to use the switch M1 for a suitable overflow path of saturated photoelectrons and integrate overflowed photoelectrons in FD and CS during a charge integration period. The non-saturated photoelectrons are transferred from PD to FD and converted to the voltage as a high sensitivity low light signal (S1) as well as the conventional 4 transistors type CMOS image sensor. The dynamic range is extended by fully utilizing the photoelectrons integrated at and overflowed from PD for a bright light signal (S2).

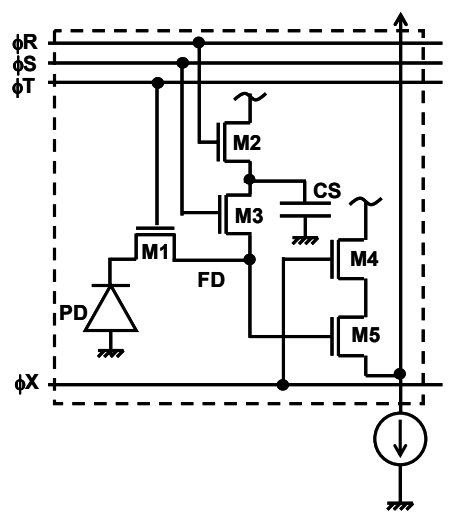

Fig.1 Pixel schematic of wide dynamic range (WDR) CMOS image sensor. 
One of the advantages in this CMOS image sensor is high tolerance to reset noise and dark current in the signal S2 [4]. Since the signal S2 is a mixture of the non-saturated and the saturated overflow photoelectrons, the minimum number of photoelectrons in S2 is close to the number of the saturated photoelectrons in $\mathrm{S} 1$ for the case of S2 selected. Precisely speaking, the only N2 in the next frame (defined as N2') can be stored in the same horizontal blanking period, however the fixed pattern noise is removed by the subtraction of (S2+N2)-N2' in the same horizontal blanking period and a high $\mathrm{S} / \mathrm{N}$ ratio can be realized in the region of $\mathrm{S} 1 / \mathrm{S} 2$ switch. This noise reduction method offers the simple operation without the necessity of the off-chip frame memory.

In order to prove the shrink-ability of the WDR pixel concept, the pixel pitch is reduced from $7.5 \mu \mathrm{m}$ (in the previous work) to $5.6 \mu \mathrm{m}$ in this work. Fig. 2 shows the chip micrograph of the $1 / 4$ inch VGA 5.6 $\mu$ m-pixel-pitch WDR-CMOS image sensor fabricated through a $0.18 \mu \mathrm{m}$ 2P3M process.

\section{Operation Temperature Range Extension}

\section{Very Low Dark Current Front End of Line}

Very-low-dark-current (VLDC) approaches are implemented to the front-end of line (FEOL) of the $0.18 \mu \mathrm{m} 2 \mathrm{P} 3 \mathrm{M}$ process. These include: the reduced plasma etching damage at the transfer gate formation, the modified channel doping profile under the transfer gate, the pinned photodiode with smaller electrical field and furnace temperature process for re-crystallization. Fig.3 compares the dark current of the VLDC FEOL and the conventional flow. Dark current is reduced to 175 $\mathrm{e}^{-/ \mathrm{sec}}$-pixel at $85^{\circ} \mathrm{C}\left(25 \mathrm{e}^{-/} / \mathrm{sec}-\right.$ pixel at $\left.60^{\circ} \mathrm{C}\right)$ by the use of the VLDC FEOL. The activation energy of the dark current for VLDC FEOL is about $0.62 \mathrm{eV}$ and should be the generation dominated.

\section{Thermal Resistant On-chip Micro Lens \& Color Filter}

The chemical aspects of the thermal decomposition of the OCML/OCCF material (phenol resin) have been analyzed with a Fourier transform infrared spectrophotometer (FTIR) as shown in Fig.4. Fig.5 shows the $\mathrm{CO}_{2}\left(2362 \mathrm{~cm}^{-1}\right)$ absorbance as the result of thermal decomposition in the varied $\mathrm{O}_{2}$ concentration from $0 \%$ to $1 \%$ in $\mathrm{O}_{2} / \mathrm{N}_{2}$ mixed gases. It is also found that $\mathrm{CO}_{2}$ over $100 \mathrm{ppb}$ is detected at $222^{\circ} \mathrm{C}$ for $1 \% \mathrm{O}_{2} / \mathrm{N}_{2}, 237^{\circ} \mathrm{C}$ for $1000 \mathrm{ppm} \mathrm{O}_{2} / \mathrm{N}_{2}$ and $282^{\circ} \mathrm{C}$ for $100 \mathrm{ppm}$. No $\mathrm{CO}_{2}$ over $100 \mathrm{ppb}$ is found under $300^{\circ} \mathrm{C}$ for the case of $<10 \mathrm{ppm}$ $\mathrm{O}_{2} / \mathrm{N}_{2}$. By preventing the OCML/OCCF from oxidation, the thermal decomposition is suppressed. In order to prevent the oxidative decomposition of the OCML/OCCF, two approaches, an inorganic $\left(\mathrm{SiO}_{2} / \mathrm{SiN}\right)$ cap onto the OCML and a metal hermetic seal package with low residual oxygen concentration in $\mathrm{N}_{2}$ gas ambient are adopted. Fig. 6 shows the schematic of the inorganic cap

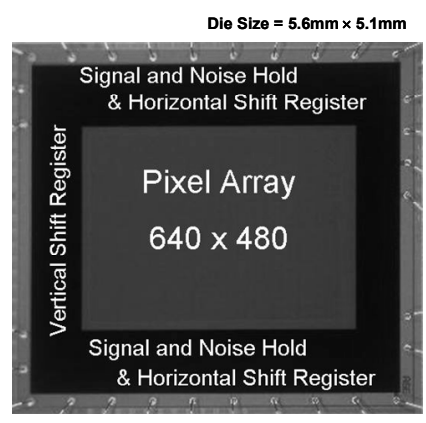

Fig.2 Chip micrograph of the $1 / 4$ inch VGA WDR CMOS image sensor with $5.6 \mu \mathrm{m}$ pixel pitch.

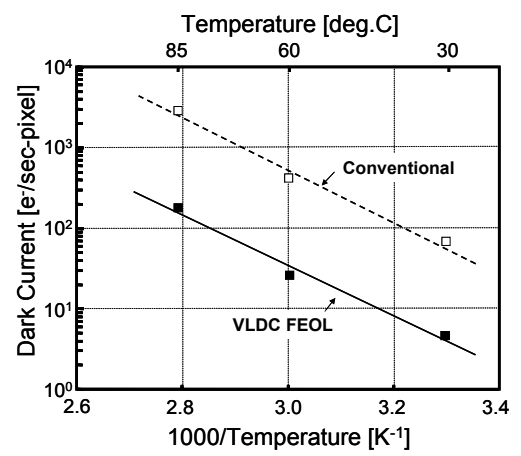

Fig.3 Dark current comparison between the VLDC FEOL and the conventional flows.

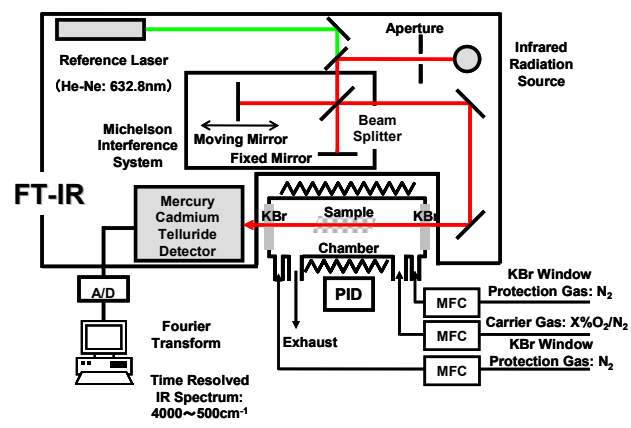

Fig.4 Schematic drawing of Fourier transform infrared spectrophotometer (FTIR).

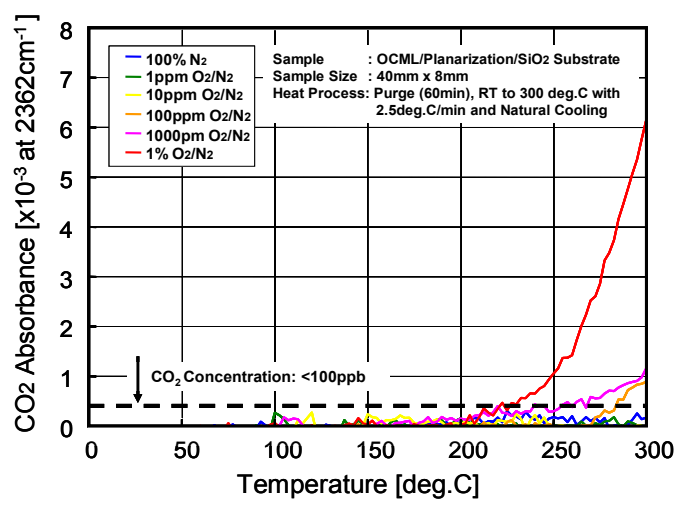

Fig.5 $\mathrm{CO}_{2}$ absorbance measured by Fourier transform infrared spectrophotometer as the result of the thermal decomposition of the micro lens in $\mathrm{O}_{2}(=0$ to $1 \%) / \mathrm{N}_{2}$ ambient. 
onto the OCML. In addition to the passivation effect, optical performance is taken into account to select the inorganic cap. The optical simulation results in improved quantum efficiency for the $\mathrm{SiO}_{2} / \mathrm{SiN}$ cap on the OCML, as shown in Fig.7. Sensor chips with and without the inorganic cap onto the OCML were assembled into a conventional package or the metal hermetic seal package, as shown in Fig.8. They were exposed to a $150^{\circ} \mathrm{C} / 500$ hours thermal stress test in the air. Fig. 9 shows the sensitivities of $\mathrm{R} / \mathrm{G} / \mathrm{B}$ pixels under a certain illumination, tested every 100 hours. The sensor chip under the thermal stress with the conventional package shows a drastic drop of sensitivity, especially in $\mathrm{B} / \mathrm{G}$ pixels. However, the other two samples (the inorganic cap onto the OCML and the metal hermetic-sealed package) show no significant degradation of the spectral response in any of $R / G / B$ pixels. The surface of the OCML is found to be protected from oxidative decomposition in both cases.

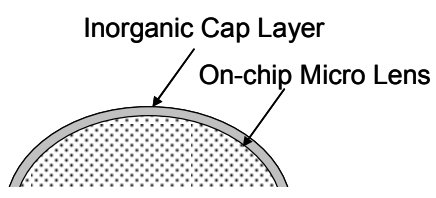

$$
\begin{aligned}
& \text { - No Cap } \\
& \text { - Inorganic Cap Layer: } \mathrm{SiO}_{2} / \mathrm{SiN}
\end{aligned}
$$

Fig.6 Inorganic cap layer onto on-chip micro lens.

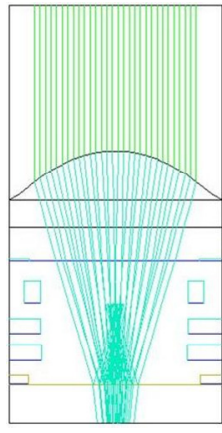

- No Cap

QE Ratio: 1.00

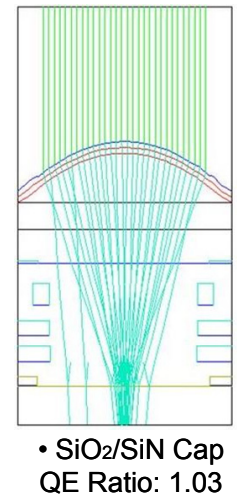

Fig.7 Optical performance of the inorganic cap layer to on-chip micro lens.

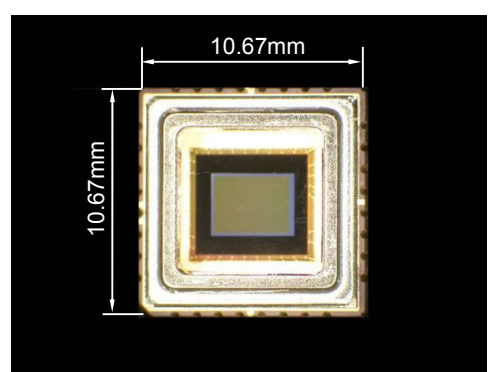

Fig.8 The WDR CMOS image sensor with the metal hermetic seal package.

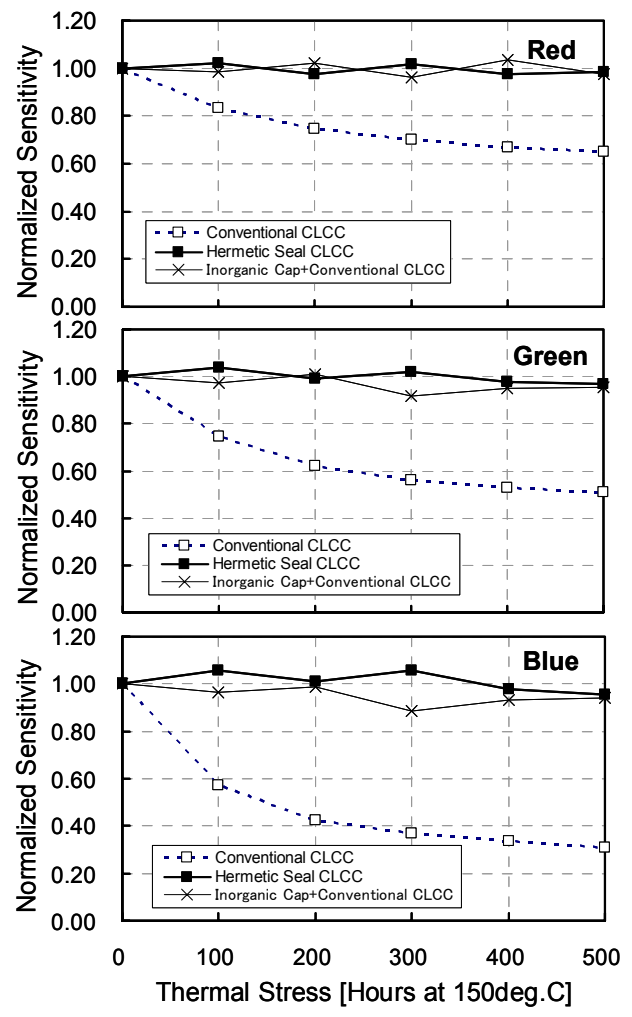

Fig.9 R, G and B sensitivities of the WDR CMOS image sensors with the hermetic seal package and the conventional package after the thermal stress test.

\section{Image Sensing Performance}

Fig.10 shows image samples captured by the temperature-resistant WDR CMOS image sensor with the metal hermetic seal package at 30,60 and $85^{\circ} \mathrm{C}$ before and after the thermal stress $\left(150^{\circ} \mathrm{C} / 500\right.$ hours $)$. The WDR signal selecting either the non-saturated signal (S1) or the saturated overflow signal (S2) by pixel is reproduced and displayed as 8 bit resolution by applying the gamma correction $(\gamma \cong 0.15)$ to 16 bit resolution data. The random noise is found to slightly increase as the temperature goes up, however no significant degradation of the image quality is observed even at $85^{\circ} \mathrm{C}$. Table 1 summarizes the image sensing performance of the temperature-resistant WDR CMOS image sensor with the metal hermetic seal package after $150^{\circ} \mathrm{C} / 500$ hours thermal stress test. The noise is $0.19 \mathrm{mV}$-rms, the effective saturation voltage of $\mathrm{S} 2$ is $8.8 \mathrm{~V}$ and the dynamic range is extended to $93 \mathrm{~dB}$. The use of the metal hermetic package prevents the oxidative decomposition of the OCML/OCCF and leads to being thermally stable even after the thermal stress test. 


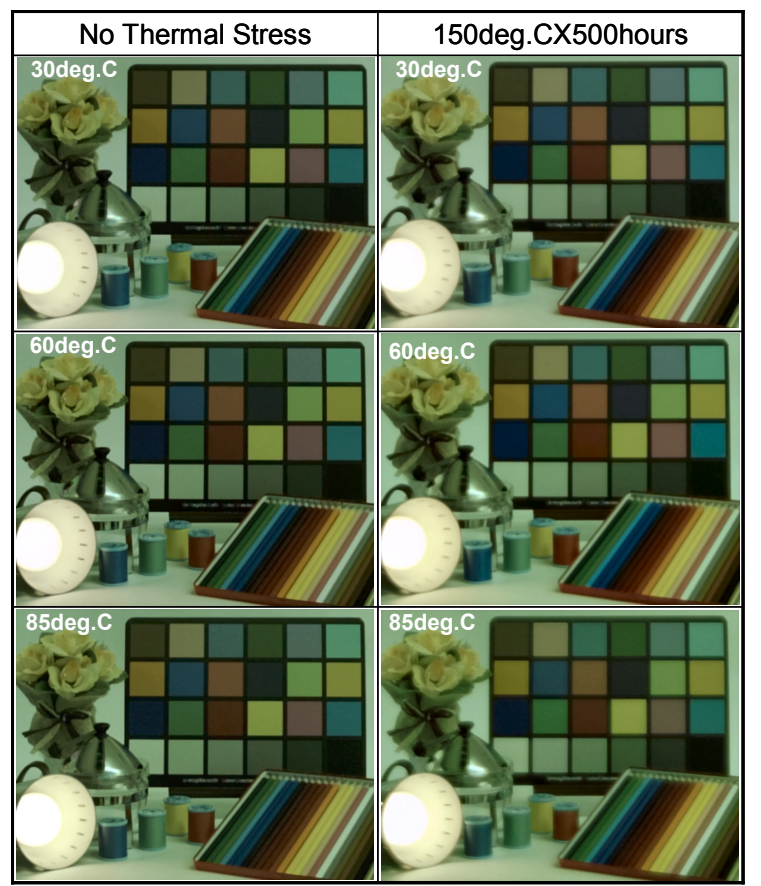

Fig.10 Image samples captured by no thermal stressed and $150^{\circ} \mathrm{C} / 500$ hours thermal stressed WDR CMOS image sensors with metal hermetic seal packages, at 30,60 and $85^{\circ} \mathrm{C}$.

Table 1 Image sensing performance of the temperatureresistant WDR CMOS image sensor with the metal hermetic seal package (: after $150^{\circ} \mathrm{C} / 500$ hours).

\begin{tabular}{|l|c|}
\hline Process technology & $0.18 \mu \mathrm{m} \mathrm{2P3M} \mathrm{CMOS}$ \\
\hline Optical format & $1 / 4$ inch \\
\hline Pixel size & $5.6 \times 5.6 \mu \mathrm{m}^{2}$ \\
\hline Number of effective pixels & $640 \times 480$ \\
\hline Supply voltage & $5 \mathrm{~V}$ \\
\hline Frame rate & $30 \mathrm{fps}$ \\
\hline Random Noise & $0.19 \mathrm{mV} \mathrm{rms}_{\mathrm{ms}}$ \\
\hline Saturation of S1 & $700 \mathrm{mV}$ \\
\hline Saturation of S2 & $1100 \mathrm{mV}$ \\
\hline $\begin{array}{l}\text { Effective saturation of S2 } \\
\text { (saturation of S2 } 8 \text { ) }\end{array}$ & $8.8 \mathrm{~V}$ \\
\hline Dynamic range & $93 \mathrm{~dB}$ \\
\hline
\end{tabular}

\section{Conclusion}

The $1 / 4$ inch VGA $(5.6 \mu \mathrm{m}$ pixel pitch) wide dynamic range (WDR) CMOS image sensor with resistance to high temperatures has been developed. The use of the very low dark current front-end of line (VLDC FEOL), the inorganic cap to the on-chip micro lens \& color filter (OCML/OCCF) and the metal hermetic seal package enables the operation temperatures up to $85^{\circ} \mathrm{C}$. The VLDC approaches which include: reduced plasma etching damage at the transfer gate formation, modified channel doping profile under the transfer gate, pinned photodiode with smaller electrical field and furnace temperature process for re-crystallization achieves $175 \mathrm{e}$-/sec-pixel of dark current at $85^{\circ} \mathrm{C}\left(25 \mathrm{e}^{-} / \mathrm{sec}-\right.$ pixel at $\left.60^{\circ} \mathrm{C}\right)$. The oxidative decomposition of the OCML/OCCF is completely suppressed, thus no significant degradation of the spectra response in any of R/G/B pixels is observed after the thermal stress test. The sample images captured by the WDR CMOS image sensor with and without the thermal stress test show no significant change in the image quality and keep a good noise performance up to 85 ${ }^{\circ} \mathrm{C}$. The dynamic range is extended to $93 \mathrm{~dB}$ even after the thermal stress test.

\section{References}

[1] S. Sugawa, N. Akahane, S. Adachi, K. Mori, T. Ishiuchi and K. Mizobuchi, "A 100 dB Dynamic Range CMOS Image Sensor Using a Lateral Overflow Integration Capacitor," 2005 ISSCC Dig. Tech. Papers, 352-353 (2005).

[2] N. Akahane, S. Sugawa, S. Adachi, K. Mori, T. Ishiuchi and K. Mizobuchi, "A Sensitivity and Linearity Improvement of a 100-dB Dynamic Range CMOS Image Sensor Using a Lateral Overflow Integration Capacitor," IEEE J. Solid-State Circuits, 41(4), 851-858 (2006).

[3] N. Akahane, R. Ryuzaki, S. Adachi, K. Mizobuchi and S. Sugawa, "A 200dB Dynamic Range Iris-less CMOS Image Sensor with Lateral Overflow Integration Capacitor using Hybrid Voltage and Current Readout Operation," 2006 ISSCC Dig. Tech. Papers, 300-301 (2006).

[4] S. Adachi, S. Sugawa, N. Akahane, K. Mori, T. Ishiuchi and K. Mizobuchi, "The Tolerance for FD Dark Current and PD Overflow Current Characteristics of Wide Dynamic Range CMOS Image Sensor Using a Lateral Overflow Integration Capacitor," Proc. 2005 IEEE Workshop on CCDs and AISs, 153-156 (2005).

[5] H. Takahashi, M. Kinoshita, K. Morita, T. Shirai, T. Sato, T. Kimura, H. Yuzurihara and S. Inoue, "A 3.9-um Pixel Pitch VGA Format 10-b Digital Output CMOS Image Sensor with 1.5 Transistor/Pixel," IEEE Journal of Solid-State Circuits, 39(12), 2417-2425 (2004).

[6] B. Pain, T. Cunningham, B. Hancok, C. Wrigley and C. Sun, "Excess Noise and Dark Current Mechanisms in CMOS Imagers," Proc. 2005 IEEE Workshop on CCDs and AISs, 145-148 (2005).

[7] K. Mizobuchi, S. Adachi, T. Yamashita, S. Okamura, H. Oshikubo, N. Akahane, S. Sugawa. "A Temperature Resistant Wide Dynamic Range CMOS Image Sensor," Proc. 2007 Electronic Imaging Science and Technology, Paper No.2501-24, to be published. 